Paul Portner, Klaus von Heusinger and Claudia Maienborn (Eds.)

Semantics - Noun Phrases and Verb Phrases 
This volume is part of a larger set of handbooks to Semantics

1 Semantics: Foundations, History and Methods

Klaus von Heusinger, Claudia Maienborn, Paul Portner (eds.)

2 Semantics: Lexical Structures and Adjectives

Claudia Maienborn, Klaus von Heusinger, Paul Portner (eds.)

3 Semantics: Theories

Claudia Maienborn, Klaus von Heusinger, Paul Portner (eds.)

$4 \quad$ Semantics: Noun Phrases and Verb Phrases

Paul Portner, Klaus von Heusinger, Claudia Maienborn (eds.)

5 Semantics: Sentence and Information Structure

Paul Portner, Claudia Maienborn, Klaus von Heusinger (eds.)

6 Semantics: Interfaces

Claudia Maienborn, Klaus von Heusinger, Paul Portner (eds.)

7 Semantics: Typology, Diachrony and Processing

Klaus von Heusinger, Claudia Maienborn, Paul Portner (eds.) 


\section{Semantics \\ Noun Phrases and Verb Phrases}

Edited by

Paul Portner

Klaus von Heusinger

Claudia Maienborn 
ISBN 978-3-11-058721-0

e-ISBN (PDF) 978-3-11-058944-3

e-ISBN (EPUB) 978-3-11-058730-2

\section{Library of Congress Cataloging-in-Publication Data}

Names: Portner, Paul, editor. | Heusinger, Klaus von, editor. | Maienborn, Claudia, editor.

Title: Semantics : noun phrases, verb phrases and adjectives / edited by Paul Portner, Klaus von Heusinger, Claudia Maienborn.

Description: Berlin ; Boston : De Gruyter, [2019] | Series: Mouton reader |

Includes bibliographical references and index.

Identifiers: LCCN 2018031262| ISBN 9783110587210 (print) | ISBN 9783110589443

(e-book (pdf) | ISBN 9783110587302 (e-book (epub)

Subjects: LCSH: Semantics. | Grammar, Comparative and

general--Grammaticalization.

Classification: LCC P325 .S37996 2019 | DDC 401/.43--dc23 LC record available at https://lccn.loc.gov/2018031262

\section{Bibliographic information published by the Deutsche Nationalbibliothek}

The Deutsche Nationalbibliothek lists this publication in the Deutsche Nationalbibliografie; detailed bibliographic data are available in the Internet at http://dnb.dnb.de.

(C) 2019 Walter de Gruyter GmbH, Berlin/Boston

Cover image: /iStock / Getty Images Plus

Typesetting: Integra Software Services Pvt. Ltd.

Printing and binding: $\mathrm{CPI}$ books $\mathrm{GmbH}$, Leck

www.degruyter.com 